\title{
AN EQUAL-AREA METHOD FOR SCALAR CONSERVATION LAWS
}

\author{
MARJETA KRAMAR FIJAVŽ ${ }^{1}$, MITJA LAKNER ${ }^{1}$ and MARJETA ŠKAPIN RUGELJ ${ }^{\otimes} 1$
}

(Received 12 November, 2011; revised 4 March, 2012)

\begin{abstract}
We study the one-dimensional conservation law. We use a characteristic surface to define a class of functions, within which the integral version of the conservation law is solved in a simple and direct way. A simple algorithm for computing the unique solution is developed. The method uses the equal-area principle and yields the solution for any given time directly.
\end{abstract}

2010 Mathematics subject classification: primary 35L65; secondary 65M25.

Keywords and phrases: conservation law, equal area, characteristics, mesh free.

\section{Introduction}

We are concerned with an important class of homogeneous hyperbolic differential equations called conservation laws, which state that measurable quantities in an isolated physical system do not change with time. We restrict ourselves to the scalar case, where the equations are of the form

$$
u_{t}+f(u)_{x}=0
$$

with $u: \mathbb{R} \times[0, \infty) \rightarrow \mathbb{R}$ representing the conserved quantity and $f: \mathbb{R} \rightarrow \mathbb{R}$ the $f$ lux. We equip equation (1.1) with the initial condition

$$
u(x, 0)=h(x), \quad x \in \mathbb{R} .
$$

In fluid mechanics, equation (1.1) with $f(u)=u v$ is called the equation of continuity and represents the conservation of mass in the motion of an ideal nonviscous fluid with mass density $u$ and velocity $v$. For the derivation and many further applications of the equation we refer to the book by Landau and Lifshitz [8]. Apart from fluid dynamics, this equation is used in various other models for the evolution of continuum quantities, such as chemical plug flow reactors or population dynamics. Here we mention only the Lighthill-Whitham-Richards model $[13,15]$, which is widely used to describe

\footnotetext{
${ }^{1}$ Faculty of Civil and Geodetic Engineering, University of Ljubljana, Jamova 2, 1000 Ljubljana, Slovenia; e-mail: mkramar@fgg.uni-lj.si, mlakner@fgg.uni-lj.si, mskapin@fgg.uni-lj.si. (C) Australian Mathematical Society 2012, Serial-fee code 1446-1811/2012 \$16.00
} 
vehicular traffic flow $[4,10,14,19]$. A very recent application of this model is given by Cristiani et al. [2], who compare the model to experimental data and describe an algorithm for making traffic forecasts.

Let us point out that although the restriction to one-dimensional problems may seem oversimplified for practical purposes, many complex problems can be reduced to onedimensional subproblems. Important examples include flows on networks, such as those treated by Cristiani et al. [2] and Gravello and Piccoli [4]; some further examples are given by Farjoun and Seibold [3, Section 8]. We believe that our approach can be very useful in tackling these problems, since it yields a precise solution for any given time.

It is well known that classical (continuously differentiable) solutions of (1.1)-(1.2), even for smooth initial conditions, do not always exist. In order to allow singularities that are meaningful for the physical problem behind the equation, one has to generalize the concept of a solution. The basic idea is to formulate a new extended problem whose continuously differentiable solutions are the classical solutions to the original equation (1.1).

We first mention the most common definition of a generalized solution. A locally $L^{1}$ function $u$ on $\mathbb{R} \times[0, \infty)$ is called a weak solution to (1.1)-(1.2) if

$$
\int_{t \geq 0} d t \int_{\mathbb{R}}\left(u \cdot \psi_{t}+f(u) \cdot \psi_{x}\right) d x+\int_{\mathbb{R}} h(x) \cdot \psi(x, 0) d x=0
$$

for every $C^{1}$ function $\psi$ on $\mathbb{R} \times[0, \infty)$ with compact support. Weak solutions are not unique, and in order to obtain the physically correct solution one has to impose the right entropy condition. There is a rich mathematical theory on this topic [1,9-11, 14].

We proceed more directly. In order to allow discontinuities, we consider the integral form of the conservation law,

$$
\frac{d}{d t} \int_{a}^{b} u(x, t) d x=f(u(a, t))-f(u(b, t))
$$

for all $a<b$ and $t>0$ for which $u$ is continuous at $(a, t)$ and $(b, t)$. This says that the area $\int_{a}^{b} u(x, t) d x$ changes in time according to the flux at the boundaries. If both $u$ and $f$ are continuously differentiable, (1.4) implies (1.1). Mathematically, the integral forms (1.3) and (1.4) are equivalent [10, p. 28]. We choose the latter because of its direct interpretation in terms of areas.

We search for solutions of (1.4) inside a class of functions $\Upsilon$ that is defined by a characteristic surface associated with the problem (1.1)-(1.2). These functions may have jumps along some locally smooth curves in the $(x, t)$-plane (see Definition 2.2). In Section 2 we prove uniqueness of solutions to (1.4) within the class $\Upsilon$. Taking the integral over all $\mathbb{R},(1.4)$ implies that

$$
\int_{\mathbb{R}} u(x, t) d x=\int_{\mathbb{R}} h(x) d x \text { for all } t \geq 0 .
$$

Hence the area under the graph of the solution does not change in time. Based on this observation, we propose in Section 3 a simple method which we call an 
equal-area method. We show that, under suitable conditions, this method yields the unique solution of (1.4) within the class $\Upsilon$.

The idea of using the equal-area principle is not new. It has already been exploited in the classical textbook by Whitham [19, Sections 2.8-2.9]. However, the method there is developed only analytically in a complicated way that is not suitable for explicit computations. Recently, Farjoun and Seibold [3] suggested a conservative particle method that utilizes the equal-area principle. They use the Lagrangian approach, representing the solution as a cloud of particles that move with the flow. Particles carry function values and move according to their characteristic velocities. When the characteristic curves collide, the particles are merged in such a way that the total area under the function is conserved. So far, we are not aware of any other numerical method that uses the equal-area principle.

Let us mention here some classical numerical schemes for conservation laws. Finite volume methods [11] are based on the integral form (1.4), instead of the differential equation (1.1), where the domain is divided into a set of grid cells. The numerical solution is an approximation of the average value of the true solution in each grid cell. One of the classical examples of finite volume methods is the Godunov scheme [5], which is based on the solution of Riemann problems.

Most numerical methods work well in the interiors of the regions of smoothness. For solving problems with discontinuities, shock tracking or front tracking [7] methods were developed. These combine standard finite difference or finite volume methods in regions of smoothness with an explicit procedure for tracking the locations of discontinuities. Shock-capturing methods use a different approach, where the goal is to capture discontinuities in the solution automatically, without explicitly tracking them. One modern shock-capturing method is a high-resolution flux-limiter Godunovtype method based on solving one-dimensional Riemann problems, described in detail by LeVeque [11] and implemented in the software package Clawpack [12].

In Section 4 we describe a numerical algorithm that we used to implement the equalarea method. We discuss numerical results obtained by our method in Section 5, and we compare our method with the aforementioned finite volume method Clawpack as well as with the newly proposed conservative particle method Particleclaw $[3,18]$. As demonstrated in Section 5, our method performs favourably in terms of efficiency and accuracy; in contrast to other known methods, where the solution at a selected time is obtained by evolution from initial conditions, it gives the solution for any given time directly. We stress, however, that the main purpose of this paper is to present theoretical principles and results; the numerical method presented aims to demonstrate the proposed algorithm, but does not claim necessarily to be the most efficient one.

\section{The characteristic surface and integral solutions}

A well-known method for treating the initial value problem (1.1)-(1.2) is the method of characteristics. Characteristics are curves in the $(x, t)$-plane along which 


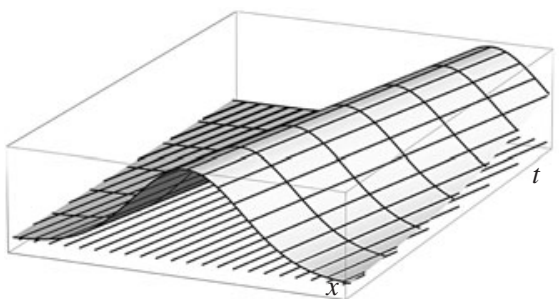

(a) Parallel characteristics

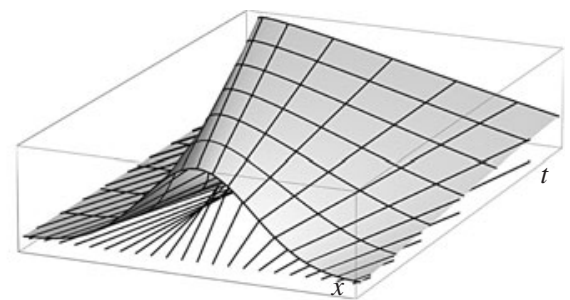

(b) Characteristics collide

FIGURE 1. Examples of characteristic surfaces $\Gamma$.

the function $u$ is constant. In our case, they are lines of the form

$$
x_{\xi}(t)=\xi+f^{\prime}(h(\xi)) t, \quad \xi \in \mathbb{R}
$$

(see, for example, the book by Logan [14, Section 2.2]). If a $C^{1}$ solution $u(x, t)$ of (1.1)-(1.2) exists, its graph in $\mathbb{R}^{3}$ is given by

$$
\Gamma:=\left\{(x, t, y)=\left(\xi+f^{\prime}(h(\xi)) t, t, h(\xi)\right) \mid \xi \in \mathbb{R}, t \geq 0\right\} .
$$

We call $\Gamma$ the characteristic surface for the problem (1.1)-(1.2). An example of such a surface is shown in Figure 1(a).

We can form $\Gamma$ a priori, before investigating the solvability of (1.1)-(1.2), so it could happen that $\Gamma$ represents a multivalued function which cannot be the solution to our problem. Indeed, this occurs whenever the characteristics collide in the $(x, t)$-plane; see Figure 1(b). In this case, the proper solution has jumps along some curves in the $(x, t)$-plane. Its graph, however, is still a subset of $\Gamma$. Starting with $\Gamma$, the solution can therefore be obtained by finding the appropriate positions of the jumps.

In the case where the initial function $h$ is not continuous, the characteristic surface $\Gamma$ defined above is not connected. Hence, before proceeding, we modify the definition of characteristic surface to correct this. For fixed $t \geq 0$ we define a plane transformation $G_{t}$ by

$$
G_{t}(x, y):=\left(x+f^{\prime}(y) t, y\right) .
$$

Denote by $\gamma_{0}$ the graph of the initial function $h$ together with the vertical line segments joining discontinuities, and define

$$
\gamma_{t}:=G_{t}\left(\gamma_{0}\right)
$$

which is a continuous curve for all $t \geq 0$; see Figure 2 .

Definition 2.1. The bounding characteristic surface for the problem (1.1)-(1.2) is

$$
\hat{\Gamma}:=\left\{(x, t, y) \mid(x, y) \in \gamma_{t}, t \geq 0\right\} .
$$



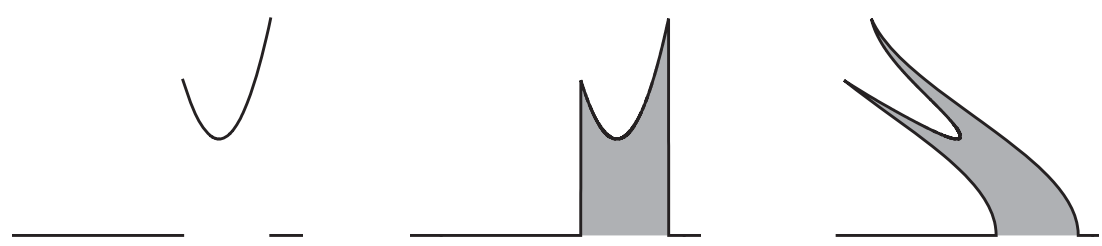

FIGURE 2. The graph of $h$ and the curves $\gamma_{0}$ and $\gamma_{t}$.

Note that $\hat{\Gamma}$ contains the characteristic surface $\Gamma$ and that the two surfaces agree whenever $h$ is continuous.

We are now ready to define the appropriate class for our solutions.

Definition 2.2. Let $f \in C^{2}(\mathbb{R})$ and let $h$ be a piecewise- $C^{1}$ function with compact support. We say that $u \in \Upsilon=\Upsilon(f, h)$ if the following conditions are satisfied:

(i) the function $u=u(x, t)$ is defined everywhere on $\mathbb{R} \times[0, \infty)$;

(ii) $u(x, 0)=h(x)$ for all $x \in \mathbb{R}$;

(iii) the graph of $u$ in $\mathbb{R}^{3}$ is a subset of the bounding characteristic surface $\hat{\Gamma}$ defined by $f$ and $h$;

(iv) the boundary of the graph of $u$ is a finite union of $C^{1}$ curves, and the projections of these curves onto the $(x, t)$-plane intersect any line $t=t_{0}$ only finitely many times;

(v) the integral $\int_{-\infty}^{\infty} u(x, t) d x$ is a continuous function of $t$ for $t \geq 0$.

Our aim is to find the solutions to the integral form of the conservation law (1.4) within the class $\Upsilon$. First, we demonstrate that every $u \in \Upsilon$ solves (1.1) on its regions of smoothness.

Lemma 2.3. Let $u \in \Upsilon$ be a $C^{1}$ function on an open set $D \subseteq \mathbb{R} \times[0, \infty)$. Then $u$ is $a$ solution of (1.1) on D.

Proof. If the graph of $u(D)$ lies on the original characteristic surface $\Gamma$, then $u$ solves (1.1) on $D$ by the method of characteristics (see the book by Lax [9, Section 3]). Therefore, we may assume that the graph of $u(D)$ is contained in $\hat{\Gamma} \backslash \Gamma$.

We show that the added vertical lines and their convolutions in time also yield a solution, in a similar way to the original characteristics. For any fixed $\xi$ we parameterize the complemented surface $\hat{\Gamma} \backslash \Gamma$ as

$$
(x, t, u(x, t))=\left(\xi+f^{\prime}(\tau) t, t, \tau\right) .
$$

Now, by implicit differentiation of the $x$-coordinate, the equality $\tau=\tau(x, t)=u(x, t)$ and the implicit function theorem [16, Theorem 9.28], one obtains

$$
u_{x}=\frac{1}{f^{\prime \prime}(\tau) t} \quad \text { and } \quad u_{t}=-\frac{f^{\prime}(\tau)}{f^{\prime \prime}(\tau) t},
$$

and one can see that $u$, implicitly defined in this way, is a local solution of (1.1). 
If, however, $u \in \Upsilon$ is not smooth for some $t>0$ and $x \in \mathbb{R}$, then it has discontinuities, called shocks, which are positioned along piecewise-smooth curves $x=s(t)$ in the $(x, t)$-plane, called shock paths. By Definition 2.2(iv), there are finitely many shock paths, which are all locally smooth. These paths may have singular points; they may cross or collide. However, we can exclude these singularities without loss of generality.

We now continue our treatment by showing that the well-known Rankine-Hugoniot condition holds in our setting.

Lemma 2.4. A function $u \in \Upsilon$ is a solution of (1.4) if and only if the following RankineHugoniot condition is satisfied at all the shocks:

$$
s^{\prime}(t)=\frac{f\left(u^{+}\right)-f\left(u^{-}\right)}{u^{+}-u^{-}} .
$$

Here $u^{+}$and $u^{-}$denote the one-sided limits of the solution $u(x, t)$ from the left and from the right of the shock, respectively; that is, $u^{+}\left(x_{0}, t\right)=\lim _{x \searrow x_{0}} u(x, t)$, with the analogous definition for $u^{-}$.

Proof. For the sake of simplicity, we omit the arguments of functions whenever they are clear from the context. Take any $a<b$. If $u \in \Upsilon$ is smooth for $x \in(a, b)$ and $t>0$, it solves $(1.1)$ on $(a, b)$ and we have

$$
\frac{d}{d t} \int_{a}^{b} u d x=\int_{a}^{b} u_{t} d x=-\int_{a}^{b} f(u)_{x} d x=f(u(a))-f(u(b)) .
$$

Now assume that $u$ has a shock on $(a, b) \times\{t\}$ with shock path $x=s(t)$. From Definition 2.2(iv), it follows that $x=s(t)$ is a locally $C^{1}$ function. Hence

$$
\begin{aligned}
\frac{d}{d t} \int_{a}^{b} u d x & =\frac{d}{d t} \int_{a}^{s(t)} u d x+\frac{d}{d t} \int_{s(t)}^{b} u d x \\
& =\int_{a}^{s(t)} u_{t} d x+s^{\prime}(t) u^{-}+\int_{s(t)}^{b} u_{t} d x-s^{\prime}(t) u^{+} \\
& =f(u(a))-f\left(u^{-}\right)+f\left(u^{+}\right)-f(u(b))+s^{\prime}(t)\left(u^{-}-u^{+}\right) .
\end{aligned}
$$

Thus, $u$ is a solution of (1.4) on $(a, b)$ if and only if the Rankine-Hugoniot condition (2.4) is fulfilled at the shock. If $u$ has more than one shock on $(a, b) \times\{t\}$, we divide the interval according to the shocks and proceed in the same manner. (Recall that Definition 2.2(iv) implies that $u$ has only finitely many shocks.)

Lax [9, Theorem 3.4] proved uniqueness of the so-called piecewise generalized solutions to (1.1). We modify slightly the idea used in his proof to show uniqueness of the solutions in our case.

Proposition 2.5. Let $f \in C^{2}(\mathbb{R})$ be strictly concave. Let $u, v \in \Upsilon$ be two solutions of (1.4) with the following properties: 
(i) $u^{-}<u^{+}$and $v^{-}<v^{+}$at every shock for $u$ and $v$, respectively;

(ii) $u(x, 0)=v(x, 0)$ for all $x \in \mathbb{R}$.

Then

$$
\|u(\cdot, t)-v(\cdot, t)\|_{1}=0 \quad \text { for all } t>0,
$$

where $\|\cdot\|_{1}$ is the $L^{1}$-norm.

Proof. Let $u, v \in \Upsilon$ be two solutions to (1.4) with $u(x, 0)=v(x, 0)=h(x)$ for all $x \in \mathbb{R}$. Since they both lie on the same characteristic surface, their difference $u-v$ can change sign only at the shocks of either (or both) of these two functions. Denote these signchanging curves in the $(x, t)$-plane by $y_{k}(t)$, and order them as

$$
y_{1}(t)<y_{2}(t)<\cdots<y_{n+1}(t) .
$$

We work on maximal open intervals of $t$ where the curves $y_{k}(t)$ do not intersect and the number of these curves does not change. For almost every $t \geq 0$ we can then write

$$
\|u(\cdot, t)-v(\cdot, t)\|_{1}=\sum_{k=1}^{n} p_{k}(t) \quad \text { where } p_{k}(t)=\int_{y_{k}(t)}^{y_{k+1}(t)}|u(x, t)-v(x, t)| d x .
$$

Now choose any interval $\left(y_{k}(t), y_{k+1}(t)\right)$ with $1 \leq k \leq n$. Without loss of generality we may assume that $u(x, t)>v(x, t)$ on this interval, and hence the absolute value under the integral defining $p_{k}(t)$ can be omitted. Note that $p_{k}(t)$ is a (continuous) piecewisedifferentiable function of $t$. If either $u$ or $v$ has shocks inside the interval, then the Rankine-Hugoniot condition is satisfied at each shock by Lemma 2.4. Dividing the interval according to these shocks and computing the derivative according to this division, as in (2.5), we see that the values around the shocks cancel out and only the values in $y_{k}(t)$ and $y_{k+1}(t)$ are important. Therefore, we assume from now on that neither $u$ nor $v$ has shocks inside the interval $\left(y_{k}(t), y_{k+1}(t)\right)$.

Thus we may assume that the function $p_{k}(t)$ is differentiable, and we now compute its derivative. As usual, we omit the arguments of functions whenever possible. Since $u$ and $v$ solve (1.1) inside the interval and since the shock paths are piecewise differentiable,

$$
\begin{aligned}
p_{k}^{\prime}(t)= & \int_{y_{k}}^{y_{k+1}}\left(u_{t}-v_{t}\right) d x+\left(u^{-}\left(y_{k+1}\right)-v^{-}\left(y_{k+1}\right)\right) y_{k+1}^{\prime}-\left(u^{+}\left(y_{k}\right)-v^{+}\left(y_{k}\right)\right) y_{k}^{\prime} \\
= & f\left(u^{+}\left(y_{k}\right)\right)-f\left(v^{+}\left(y_{k}\right)\right)-\left(u^{+}\left(y_{k}\right)-v^{+}\left(y_{k}\right)\right) y_{k}^{\prime} \\
& -\left[f\left(u^{-}\left(y_{k+1}\right)\right)-f\left(v^{-}\left(y_{k+1}\right)\right)-\left(u^{-}\left(y_{k+1}\right)-v^{-}\left(y_{k+1}\right)\right) y_{k+1}^{\prime}\right] .
\end{aligned}
$$

From now on, we explain calculations only for the left endpoint of the interval, since the right endpoint can be treated symmetrically. By assumption, $u>v$ inside the interval, and hence $u-v$ changes sign at the endpoints and all the jumps are upwards. Taking all of this into account, we see that $u$ has a shock in $y_{k}$ while $v$ may or may not have a shock (in the latter case we take $v^{-}=v^{+}$). Furthermore,

$$
u^{-} \leq v^{-} \leq v^{+} \leq u^{+} \text {. }
$$


Applying the Rankine-Hugoniot condition for the speed of the shock, $y_{k}^{\prime}$, for $u$, we see that the expression in (2.8) is equal to

$$
\left(\frac{f\left(u^{+}\right)-f\left(v^{+}\right)}{u^{+}-v^{+}}-\frac{f\left(u^{+}\right)-f\left(u^{-}\right)}{u^{+}-u^{-}}\right)\left(u^{+}-v^{+}\right) \leq 0,
$$

since by concavity of $f$ and (2.10) the first factor is less than or equal to 0 while $u^{+}-v^{+}>0$. Following the same line of argument for the right endpoint $y_{k+1}$, we obtain the same conclusion for the expression in (2.9). We have thus shown that $p_{k}^{\prime}(t) \leq 0$ for $1 \leq k \leq n$. By $(2.7),\|u(\cdot, t)-v(\cdot, t)\|_{1}$ is then a decreasing function of $t$. Since by assumption $\|u(\cdot, 0)-v(\cdot, 0)\|_{1}=0$, we finally obtain (2.6).

We have seen that the solutions to (1.4) in the class $\Upsilon$ are unique, provided that the flux is a concave function and the solutions only have upward jumps. Note that the same is true for convex flux and downward jumps.

REMARK 2.6. Using Lemmas 2.3 and 2.4, we see that our solutions in the class $\Upsilon$ are weak solutions (see also the book by Bressan [1, Theorem 4.2]). Condition (i) of Proposition 2.5 is actually an entropy condition which yields the physically reasonable solution (see the books by Lax [9, equation (3.13)], LeVeque [10, p. 36] and Bressan [1, equation (4.38)]).

\section{The equal-area method}

We now describe the equal-area method for obtaining the solutions starting from the bounding characteristic surface $\hat{\Gamma}$ defined in (2.3). First, note that the transformation $G_{t}$ defined in (2.1) preserves area, since its Jacobian is 1 . Hence the area bounded by the curves $\gamma_{t}$ and the $x$-axis remains unchanged in time and is equal to the initial area given by $\int_{-\infty}^{\infty} h(x) d x$ (compare the shaded areas in Figure 2). Intersecting $\hat{\Gamma}$ with the plane $t=t_{0}$ for some fixed time $t_{0}$ yields $\gamma_{t_{0}}$ defined in (2.2); see also Figure 2. Our strategy is to insert vertical cuts to $\gamma_{t_{0}}$ in such a way that the areas of the cut-off lobes coincide. Thus the initial area will be preserved. Upon carrying out this procedure for all $t$, we obtain a bounded piecewise-continuous function $u(x, t)$ whose graph, without the added vertical surfaces, is contained in $\hat{\Gamma}$; see Figures 3 and 6.

Lemma 3.1. Let $f \in C^{2}(\mathbb{R})$ be strictly concave and let $h$ be a piecewise- $C^{1}$ function with compact support. Then, for the solution u obtained by the equal-area method described above, the following properties hold:

(i) at every shock, $u$ satisfies the Rankine-Hugoniot condition (2.4) and has only upward jumps, $u^{-}<u^{+}$;

(ii) the shock paths are piecewise- $C^{1}$ curves.

Proof. For $t>0$ we have the curves

$$
\gamma_{t}(\xi)=\left(x_{t}(\xi), y_{t}(\xi)\right)=\left(\xi+f^{\prime}(h(\xi)) t, h(\xi)\right), \quad \xi \in \mathbb{R}
$$




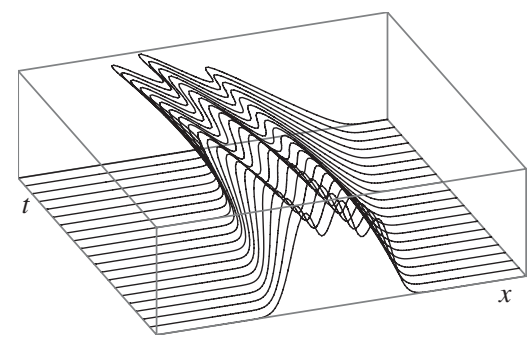

(a) The bounding characteristic surface $\hat{\Gamma}$

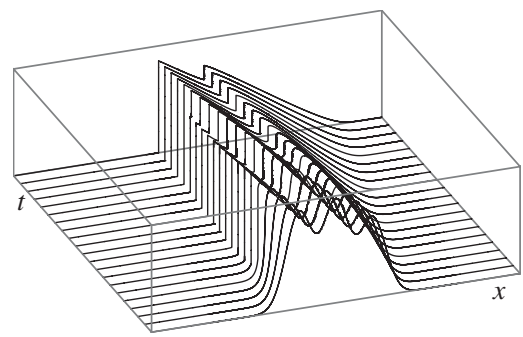

(b) The solution $u(x, t)$ (with vertical surfaces at the positions of the jumps)

FIGURE 3. Graphs for the problem (1.1)-(1.2) with the initial function given in (5.1).

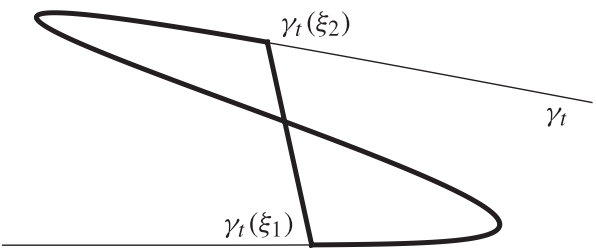

Figure 4. The " $S$-curve".

For $\xi_{1}<\xi_{2}$, we close the " $S$-curve" $\gamma_{t}\left(\left[\xi_{1}, \xi_{2}\right]\right)$ with a line segment between the endpoints $\gamma_{t}\left(\xi_{1}\right)$ and $\gamma_{t}\left(\xi_{2}\right)$; see Figure 4. By Green's theorem, the signed area defined by this closed curve is

$$
\begin{aligned}
p_{t}\left(\xi_{1}, \xi_{2}\right)= & \frac{1}{2} \int_{\xi_{1}}^{\xi_{2}}\left[x_{t}(\xi) y_{t}^{\prime}(\xi)-y_{t}(\xi) x_{t}^{\prime}(\xi)\right] d \xi \\
& +\frac{1}{2} \int_{0}^{1}\left[\left(\xi x_{t}\left(\xi_{1}\right)+(1-\xi) x_{t}\left(\xi_{2}\right)\right)\left(y_{t}\left(\xi_{1}\right)-y_{t}\left(\xi_{2}\right)\right)\right. \\
& \left.\quad-\left(\xi y_{t}\left(\xi_{1}\right)+(1-\xi) y_{t}\left(\xi_{2}\right)\right)\left(x_{t}\left(\xi_{1}\right)-x_{t}\left(\xi_{2}\right)\right)\right] d \xi .
\end{aligned}
$$

Define the mapping $F: \mathbb{R}^{2} \times \mathbb{R}^{+} \rightarrow \mathbb{R}^{2}$ by

$$
F\left(\xi_{1}, \xi_{2}, t\right):=\left(p_{t}\left(\xi_{1}, \xi_{2}\right), x_{t}\left(\xi_{1}\right)-x_{t}\left(\xi_{2}\right)\right) .
$$

At points where

$$
x_{t}\left(\xi_{1}^{0}\right)=x_{t}\left(\xi_{2}^{0}\right)
$$

the determinant of the $2 \times 2$ Jacobian matrix $\partial F / \partial \xi$ is equal to

$$
2\left(y_{t}\left(\xi_{2}^{0}\right)-y_{t}\left(\xi_{1}^{0}\right)\right) x_{t}^{\prime}\left(\xi_{1}^{0}\right) x_{t}^{\prime}\left(\xi_{2}^{0}\right),
$$

which is nonzero for jumps given by our method.

If in addition to (3.1) we have the equal-area condition $p_{t_{0}}\left(\xi_{1}^{0}, \xi_{2}^{0}\right)=0$, then the implicit function theorem [16, Theorem 9.28] gives the existence of two $C^{1}$ 
functions $\widehat{\xi}_{1}(t)$ and $\widehat{\xi}_{2}(t)$ such that for all $t$ in some neighbourhood of $t_{0}$,

$$
F\left(\widehat{\xi}_{1}(t), \widehat{\xi}_{2}(t), t\right)=(0,0), \quad \widehat{\xi}_{1}\left(t_{0}\right)=\xi_{1}^{0}, \quad \widehat{\xi}_{2}\left(t_{0}\right)=\xi_{2}^{0} .
$$

This means that the equal-area condition holds for all parameters $t$ in this neighbourhood. Moreover, the implicit function theorem gives the formula

$$
\left[\begin{array}{l}
\xi_{1}^{\prime}\left(t_{0}\right) \\
\widehat{\xi_{2}^{\prime}}\left(t_{0}\right)
\end{array}\right]=-\left(\frac{\partial F}{\partial \xi}\right)^{-1} \frac{\partial F}{\partial t} .
$$

Using symbolic computation (Mathematica), one obtains

$$
\widehat{\xi}_{1}^{\prime}\left(t_{0}\right)=\frac{f\left(h\left(\xi_{1}^{0}\right)\right)-f\left(h\left(\xi_{2}^{0}\right)\right)+\left(h\left(\xi_{2}^{0}\right)-h\left(\xi_{1}^{0}\right)\right) f^{\prime}\left(h\left(\xi_{1}^{0}\right)\right)}{\left(h\left(\xi_{1}^{0}\right)-h\left(\xi_{2}^{0}\right)\right)\left(1+f^{\prime \prime}\left(h\left(\xi_{1}^{0}\right)\right) h^{\prime}\left(\xi_{1}^{0}\right) t_{0}\right)} .
$$

By the above, the shock path

$$
x(t)=\widehat{\xi}_{1}(t)+f^{\prime}\left(h\left(\widehat{\xi}_{1}(t)\right)\right) t
$$

is locally a $C^{1}$ function, which proves (ii). Differentiating this function at the point $t=t_{0}$, we finally obtain the Rankine-Hugoniot condition (2.4):

$$
\begin{aligned}
x^{\prime}\left(t_{0}\right) & =\widehat{\xi_{1}^{\prime}}\left(t_{0}\right)+f^{\prime \prime}\left(h\left(\xi_{1}^{0}\right)\right) h^{\prime}\left(\xi_{1}^{0}\right) \widehat{\xi}_{1}^{\prime}\left(t_{0}\right) t_{0}+f^{\prime}\left(h\left(\xi_{1}^{0}\right)\right) \\
& =\frac{f\left(h\left(\xi_{2}^{0}\right)\right)-f\left(h\left(\xi_{1}^{0}\right)\right)}{h\left(\xi_{2}^{0}\right)-h\left(\xi_{1}^{0}\right)}=\frac{f\left(u^{+}\right)-f\left(u^{-}\right)}{u^{+}-u^{-}} .
\end{aligned}
$$

In the case where $f$ is concave, $f^{\prime}$ is a decreasing function and the curves $\gamma_{t}$ are inclined to the left for $t>0$ (see the last plot in Figure 2). It then follows from the construction that the solution $u$ has only upward jumps; hence assertion (i) is also proved.

We have proved the local smoothness of the shock paths, a nice property which is often assumed in advance and rarely verified. Assuming a finite number of shocks, we are finally able to prove that the equal-area method yields the unique solution to our problem.

THeOREM 3.2. Let $f \in C^{2}(\mathbb{R})$ be a strictly concave function and $h$ a piecewise- $C^{1}$ function with compact support such that the function

$$
x_{t}(\xi)=\xi+f^{\prime}(h(\xi)) t, \quad \xi \in \mathbb{R}
$$

has only finitely many local extrema for every $t \geq 0$. Then the equal-area method described above yields the unique solution $u \in \Upsilon$ to (1.4).

Proof. First, observe that conditions (i)-(iii) and (v) of Definition 2.2 are trivially fulfilled for a function $u$ obtained by the equal-area method. Since the number of local extrema of the function in (3.2) is finite, so is the number of shocks obtained by the equal-area method. Hence Definition 2.2(iv) is satisfied, by Lemma 3.1. Moreover, using Lemmas 2.3, 2.4 and 3.1 and Proposition 2.5, we see that $u \in \Upsilon$ is the unique solution of (1.4). 


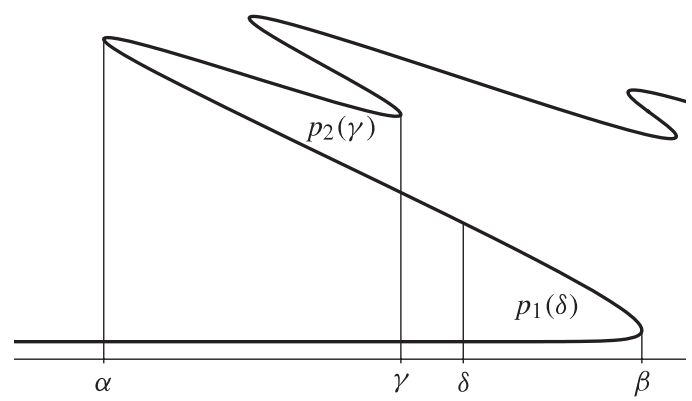

FIGURE 5. Significant points $\alpha, \beta, \gamma$ and $\delta$ on the curve $K_{i}$ and the areas $p_{1}(\delta)$ and $p_{2}(\gamma)$.

A brief comment is in order here. For our theoretical approach we need to assume that there are only finitely many shocks. We are not aware of any explicit conditions in terms of the functions $f$ and $h$ for meeting this assumption (some generic conditions are given by Golubitsky and Schaeffer $[6,17])$. In practice, however, it is not difficult to check the finiteness of the number of local extrema of the function $x_{t}(\xi)$ in (3.2) for any given $f, h$ and $t$. (Note that our procedure gives a solution for any fixed time t.) Moreover, numerically this condition is always satisfied, since we use polygonal approximation for continuous curves.

\section{The algorithm}

We now describe the algorithm based on the procedure introduced in the previous section. The solution at some fixed time $t_{0}$ is obtained directly, without the need to march forward in time. We start by taking a polygonal approximation $K_{0}$ for the continuous curve

$$
\gamma_{t_{0}}:=G_{t_{0}}\left(\gamma_{0}\right)
$$

see (2.1)-(2.2). We use points on the curve $\gamma_{t_{0}}$ obtained from equidistant parameters on the $x$-axis, $G_{t_{0}}\left(x_{i}, h\left(x_{i}\right)\right)$. Travelling along the curve, we gradually "equalize" the areas. The obtained graph of the solution is a subset of $K_{0}$ (see Figure 6).

Algorithm 4.1 (Iterative step). Let the parametrization $(x(\tau), y(\tau)), \tau \in \mathbb{R}$, of the curve $K_{i}$ at the $i$ th step be such that $x(\tau)$ is increasing on the far ends of the interval $(-\infty, \infty)$. We first define three significant points for the curve $K_{i}$ (see Figure 5):

- $\beta=x\left(\tau_{1}\right)$ is the first local maximum of $x(\tau)$ in the direction of the increasing parameter $\tau$. If such a maximum does not exist, then we are done, and $K_{i}$ is the graph of the weak solution (with redundant vertical lines in the jumps).

- $\alpha=x\left(\tau_{2}\right)$ is the first local minimum of $x(\tau)$ from $\tau_{1}$ onwards. Since the function $x(\tau)$ is not bounded from above, such a minimum always exists.

- $\gamma$ is the minimum of $\beta$ and the next local maximum of $x(\tau)$. If such a maximum does not exist, set $\gamma=\beta$. 


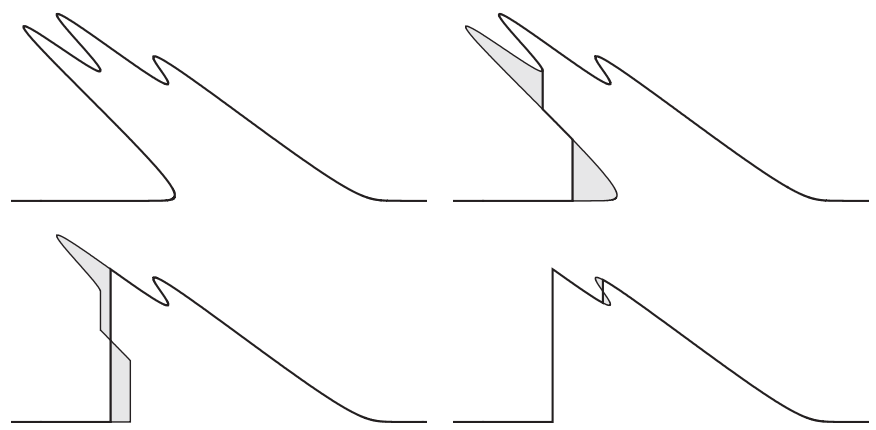

Figure 6. Curves $K_{i}, i=0,1,2,3$, obtained from three consecutive steps of Algorithm 4.1, resulting in the solution at time $t_{0}$.

We now compute the areas. For any $x_{0} \in(\alpha, \beta)$, denote by $p_{1}\left(x_{0}\right)$ the area bounded by the line $x=x_{0}$ and the part of the curve $K_{i}$ that contains $\left(x\left(\tau_{1}\right), y\left(\tau_{1}\right)\right)$. For any $x_{0} \in(\alpha, \gamma)$, denote by $p_{2}\left(x_{0}\right)$ the area bounded by the line $x=x_{0}$ and the part of the curve $K_{i}$ that contains $\left(x\left(\tau_{2}\right), y\left(\tau_{2}\right)\right)$. For $x \in(\alpha, \gamma)$, let

$$
p(x):=p_{2}(x)-p_{1}(x) \text {. }
$$

Then $p_{1}(x)$ is continuously decreasing while $p_{2}(x)$ and $p(x)$ are continuously increasing, and $p(\alpha)=0-p_{1}(\alpha)<0$. We distinguish two cases:

- If $p(\gamma) \geq 0$, let $\delta \in(\alpha, \gamma]$ be the only zero of the function $p(x)$. Then $p_{1}(\delta)=$ $p_{2}(\delta)$. The curve $K_{i+1}$ is obtained from $K_{i}$ by replacing the parts of $K_{i}$ that determine $p_{1}(\delta)$ and $p_{2}(\delta)$ with vertical lines.

- If $p(\gamma)<0$, then $p_{1}(\gamma)>p_{2}(\gamma)$ and, by continuity and monotonicity of the function $p_{1}(x)$, there exists only one $\delta \in(\gamma, \beta)$ which satisfies $p_{1}(\delta)=p_{2}(\gamma)$ (note that $\left.p_{1}(\beta)=0\right)$. The point $\delta$ and the areas $p_{1}(\delta)$ and $p_{2}(\gamma)$ are marked in Figure 5. The new curve $K_{i+1}$ is obtained from $K_{i}$ by replacing those parts of $K_{i}$ that determine $p_{1}(\delta)$ and $p_{2}(\gamma)$ with vertical lines.

Figure 6 shows an example of the results after applying three steps of the above algorithm to the function given in (5.1) at time $t_{0}=4.25$.

We briefly describe the method that we use to compute the areas needed in each step of Algorithm 4.1. Let $D$ be a polygon determined by the points $T_{1}\left(x_{1}, y_{1}\right), \ldots, T_{n}\left(x_{n}, y_{n}\right)$ (we orient these in a counterclockwise direction), and let $T_{0}\left(x_{0}, y_{0}\right)$ be any point in the plane. Then the signed area of the triangle $T_{0} T_{i} T_{i+1}$ can be computed by

$$
p_{0, i, i+1}:=\frac{1}{2}\left|\begin{array}{ll}
x_{i}-x_{0} & y_{i}-y_{0} \\
x_{i+1}-x_{0} & y_{i+1}-y_{0}
\end{array}\right| .
$$

By Green's theorem, one can see that the area of the polygon $D$ is then equal to

$$
p=\sum_{i=1}^{n} p_{0, i, i+1} \quad \text { where } T_{n+1}=T_{1} .
$$




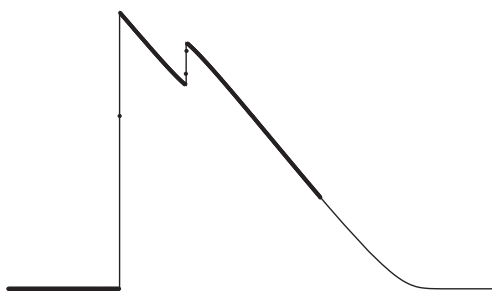

(a) Clawpack

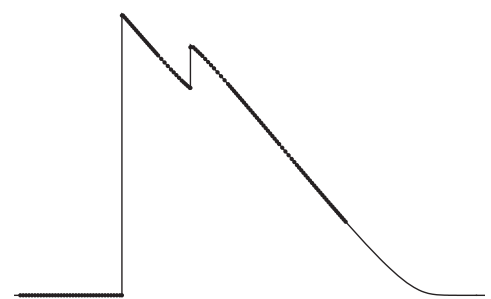

(b) Particleclaw

FIGURE 7. The solution obtained by the equal-area method (thin line) compared to the results of Clawpack and Particleclaw (thick dots).

\section{Numerical results}

We programmed our equal-area method in Mathematica and first compared the results with those obtained from the basic Godunov method (which we also implemented in Mathematica). Furthermore, we compared our method with an advanced Godunov method, which is the basis of the widely used software package Clawpack [12]. Finally, we made a comparison to the very recently developed software package Particleclaw [18], which uses a Langrangian particle method and some information about the characteristics. Both of the above software packages are freely available.

The results of these tests are very good: our algorithm performs favourably in terms of both time efficiency and accuracy. Figure 7 shows graphs of the solution obtained by our method and by the two software packages mentioned above for the initial condition

$$
h(x)= \begin{cases}0.9 e^{-x^{2}}+0.7 e^{-(x-2)^{2}}+0.85 e^{-(x+2)^{2}} & \text { if } x \in[-10,10] \\ 0 & \text { otherwise }\end{cases}
$$

and the flux function $f(u)=u(1-u)$.

The time complexity of our algorithm depends mostly on finding zeros of a function, obtained by computing areas of polygons. We used the secant method, and typically 7 to 12 iterations ( 9 on average) were needed to attain $10^{-14}$ accuracy. The number of necessary steps of Algorithm 4.1 is bounded above by the number of stationary points of the function $x_{t_{0}}(\xi)$ defined in (3.2).

We can approximate the error of the position of the shock. Assuming that the original curve $\gamma_{t_{0}}$ lies in an $\varepsilon$-neighborhood of a polygonal approximation line, we have an approximation of the area between the " $S$-curves" as in Figure 5:

$$
l \varepsilon \doteq \Delta x s,
$$

where $l$ is the length of the " $S$-curve", $\Delta x$ is the displacement of the true shock and $s$ is the height of the shock. This gives an approximation of the displacement $\Delta x$ :

$$
\Delta x \doteq \varepsilon \frac{l}{s} .
$$




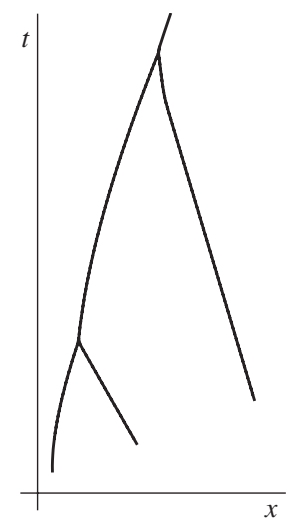

FIGURE 8. Shock paths in the $(x, t)$-plane for the initial function given in (5.1).

In Figure 6 the polygonal approximation with 1000 points has $\varepsilon$ less than $6 \times 10^{-4}$, and $\Delta x$ is approximated as $3 \times 10^{-3}$. The method is quadratic; that is, doubling the number of points would result in decreasing the value of $\varepsilon$ by a factor of 4 .

\section{Conclusions}

By using (bounding) characteristic surfaces, we defined the proper solution to the integral form of the conservation law (1.4). We proposed an equal-area method and showed that the (unique) solution obtained has all the desired properties: it solves (1.1)-(1.2) exactly wherever it is smooth, it satisfies the Rankine-Hugoniot condition (2.4) at all the shocks, and the shock paths are locally smooth. Finally, we have described an algorithm for implementing our method and compared it to some known methods.

Our equal-area method has the following advantages:

- In contrast to classical numerical schemes for conservation laws, it is mesh free.

- The method is by nature exactly conservative.

- The solution is computed for any given fixed time; hence the errors do not accumulate in time.

- The method is accurate: the quality of the approximation relies only on the quality of the starting approximation of the curve $\gamma_{t_{0}}$ with a polygonal line $K_{0}$.

- Some methods treat the rarefaction waves separately, using different techniques from those for shock waves. There is no need for such separate treatment in our case, and the rarefaction waves are created on the way where appropriate.

- The shocks obtained are sharp and propagate with the correct speed. Their position is obtained automatically by equalizing the appropriate areas. Moreover, the shock paths are obtained easily by computing the solution for some selected times and then simply projecting the shocks from the surface to the $(x, t)$-plane (see Figure 8). 


\section{Acknowledgements}

The authors would like to express their gratitude to Aleš Založnik and Marijan Žura for motivation and many helpful discussions.

\section{References}

[1] A. Bressan, Hyperbolic systems of conservation laws: the one-dimensional Cauchy problem (Oxford University Press, New York, 2005).

[2] E. Cristiani, C. de Fabritiis and B. Piccoli, "A fluid dynamic approach for traffic forecast from mobile sensor data", Comm. Appl. Ind. Math. 1 (2010) 54-71; doi:10.1685/2010CAIM487.

[3] Y. Farjoun and B. Seibold, "A rarefaction-tracking method for hyperbolic conservation laws", J. Engrg. Math. 66 (2010) 237-251; doi:10.1007/s10665-009-9338-3.

[4] M. Garavello and B. Piccoli, Traffic flow on networks (American Institute of Mathematical Sciences, Springfield, MO, 2006).

[5] S. K. Godunov, "A difference scheme for numerical solution of discontinuous solution of hydrodynamic equations", Mat. Sb. 47 (1959) 271-306.

[6] M. Golubitsky and D. G. Schaeffer, "Stability of shock waves for a single conservation law", $A d v$. Math. 16 (1975) 65-71; doi:10.1016/0001-8708(75)90100-0.

[7] H. Holden and N. H. Risebro, Front tracking for hyperbolic conservation laws (Springer, New York, 2009).

[8] L. D. Landau and E. M. Lifshitz, Fluid mechanics (Pergamon Press, Oxford, 1987).

[9] P. Lax, Hyperbolic systems of conservation laws and the mathematical theory of shock waves (Society of Industrial and Applied Mathematics, Philadelphia, 1973).

[10] R. J. LeVeque, Numerical methods for conservation laws (Birkhäuser, Basel, 1992).

[11] R. J. LeVeque, Finite-volume methods for hyperbolic problems (Cambridge University Press, Cambridge, 2004).

[12] R. J. LeVeque et al., Clawpack, http://www.clawpack.org.

[13] M. J. Lighthill and G. B. Whitham, "On kinematic waves. II. A theory of traffic flow on long crowded roads", Proc. R. Soc. Lond. A 229 (1955) 317-345; doi:10.1098/rspa.1955.0089.

[14] J. D. Logan, An introduction to nonlinear partial differential equations (John Wiley \& Sons, Hoboken, NJ, 2008).

[15] P. I. Richards, "Shock waves on the highway", Oper. Res. 4 (1956) 42-51; doi:10.1287/opre.4.1.42.

[16] W. Rudin, Principles of mathematical analysis (McGraw-Hill, Auckland, 1976).

[17] D. G. Schaeffer, "A regularity theorem for conservation laws", Adv. Math. 11 (1973) 368-386; doi:10.1016/0001-8708(73)90018-2.

[18] B. Seibold, Particleclaw, http://www.math.temple.edu/ seibold/research/particleclaw/.

[19] G. B. Whitham, Linear and nonlinear waves (John Wiley \& Sons, New York, 1974). 